\title{
Association between the XRCC3 Thr241Met Polymorphism and Risk of Colorectal Cancer: a Meta Analysis of 5,193 Cases and 6,645 Controls
}

\author{
Abolfazl Namazi ${ }^{1}$, Maryam Abedinzadeh ${ }^{2}$, Parisa Nourbaksh ${ }^{3}$, Hossein \\ Neamatzadeh $^{3}$
}

\begin{abstract}
Background: Many studies have reported associations of the X-ray repair cross-complementing group 3 (XRCC3) Thr241Met polymorphism with colorectal cancer (CRC) risk, but the results remained controversial. Hence, we performed the present meta-analysis with different inheritance models. Materials and Methods: We searched the PubMed and Google scholar databases for studies relating to associations between XRCC3 Thr241Met polymorphism and risk of CRC. 16 studies with 5,193 cases and 6,645 controls were finally included into the meta-analysis. Results: We found that the XRCC3 Thr241Met polymorphism was associated with increased CRC risk only under a dominant genetic model (CC+CT vs. TT: OR 0.575,95\% CI 0.498-1.665, p<0.001, $\left.P_{\text {heterogeneity }}=0.00, I^{2}=83 \%\right)$. There was a significant association between XRCC3 Thr241Met polymorphism and CRC risk in Caucasian in the overall 8 studies under only in the heterozygote genetic model (CT vs. TT: OR=0.929, 95\% CI =0.806-1.070, P=0.308, $P_{\text {heterogeneity }}=0.002, I^{2}=57 \%$ ). Four studies evaluated the XRCC3 Thr241Met polymorphism and CRC risk in Asians. Two genetic models of the XRCC3 polymorphism were significantly correlated with increasing risk in Asians (dominant model: $\mathrm{CC}+\mathrm{CT}$ vs. TT: $\mathrm{OR}=0.609,95 \% \mathrm{CI}=411-0.902$, $\mathrm{P}=0.013, \mathrm{P}_{\text {heterogeneity }}=0.54, \mathrm{I}^{2}=0.00 \%$; Allele model: $\mathrm{C}$ vs. $\mathrm{T}: \mathrm{OR}=0.708,95 \%=\mathrm{CI}$ 0.605-0.829, $\mathrm{p}=0.000, \mathrm{P}_{\mathrm{b}}$ $\left.=0.000, I^{2}=92 \%\right)$. The sensitivity analysis suggested stability of this meta-analysis and no publication bias was detected. Conclusions: In conclusion, this meta-analysis indicates that XRCC3 Thr241Met shows an increased CRC risk, particularly in Asians rather than Caucasians.
\end{abstract}

Keywords: Colorectal cancer - XRCC3 Thr241Met - polymorphism - Caucasians - Asians

Asian Pac J Cancer Prev, 16 (6), 2263-2268

\section{Introduction}

Worldwide, colorectal cancer (CRC) is the third most frequently diagnosed cancer in males and the second in females (Gao et al., 2014). Australia and New Zealand, Europe and North America have the highest incidence rates of CRC worldwide, and Africa and South-Central Asia, the lowest (Jemal et al., 2011). Over $75 \%$ of CRCs occur sporadically, with only $25 \%$ of patients having a family history of CRC (Mostafa et al., 2011). Epidemiological studies have demonstrated that some risk factors and interactions between genetic and environmental factors may play important roles in the pathogenesis of that cancer (Mahmoudi et al., 2014).

Genetic polymorphisms in DNA repair genes may influence individual variation in DNA repair capacity, which may be associated with a higher risk of developing cancer. X-ray repair cross-complementing group 3 gene
(XRCC3, also known as CMM6 and OMIM, 600675 is a RAD51 paralog that functions in the repair of DNA double-strand breaks (DSBs) by homologous recombination (Alanazi et al., 2013). XRCC3 mutation causes severe chromosome instability (Brenneman et al., 2002). Many studies have investigated the role of X-ray repair cross-complementing group in cancer. Abnormal expression or activity of XRCC3 observed in many types of cancer, like breast cancer, colorectal cancer, non-small-cell lung cancer, head and neck squamous cell carcinoma, has been considered as an important marker in tumorigenesis, which resulted in more aggressive tumor phenotype, higher recurrence rate, and poorer prognosis (Bewick et al., 2006; Zhan et al., 2013). Tebbs et al. mapped the XRCC3 gene to human chromosome 14q32.3 by fluorescence in situ hybridization and Southern blot hybridization with genomic DNA from 2 independent hybrid clone panels (Tebbs et al., 1995). 
A common polymorphism in exon 7 of the XRCC3 gene results in an amino acid substitution at codon 241 (Thr241Met) that may affect the enzyme function and/ or its interaction with other proteins involved in DNA damage and repair. Growing evidence suggests that the XRCC3 Thr241Met (T241M) polymorphism in the homologous recombination repair gene XRCC3 may alter DNA repair capacity and subsequent susceptibility to carcinogens. However, studies on the association between DNA repair gene polymorphisms especially XRCC3 and colorectal cancer risk appear to be very limited.

Krupa et al. (2004) reported that CRC occurrence was strongly associated with the XRCC3 Met/Met polymorphic variant $(\mathrm{OR}=9.45$; (95\%CI 8.77-11.65), whereas $\mathrm{Thr} / \mathrm{Thr}$ and $\mathrm{Thr} / \mathrm{Met}$ variants were associated with significant reduction in colorectal cancer risk $(\mathrm{OR}=0.16$; 95\% CI 0-0.26 and $\mathrm{OR}=0.26$; 95\% CI 0.25-0.27, respectively) (Krupa et al., 2004). However, other casecontrol studies, of this SNP failed to detect a significant association for CRC risk (Zhou et al., 2014). Larger sample size and well-designed studies about the XRCC3 Thr241Met association with CRC are warranted to further confirm the results.

The objective of this effort was to conduct a metaanalysis of the published data to determine whether relationships exist between the XRCC3 Thr241Met polymorphism and the incidence of CRC.

\section{Materials and Methods}

\section{Search strategy}

A literature research was conducted using PubMed, Web of Science and CNKI up to January 2015 without with language restrictions. Relevant studies were identified using the terms: "XRCC3" AND"Thr241Met" AND "genetic polymorphism or polymorphism or variant" AND "colorectal cancer or carcinoma or CRC". Additional studies were identified by a hand search of references of original or review articles on this topic. If data or data subsets were published in more than one article, only the publication with the largest sample size was included.

\section{Inclusion criteria and exclusion criteria}

Studies were included if they met the following criteria: (1) in a case-control study design, (2) studies on the relationship of XRCC3 Thr241Met polymorphism and colorectal cancer, (3) sufficient data for the frequencies of alleles and genotypes in cases and controls or could be calculated from the article text. While major exclusion criteria were: (1) case-only study, case reports, and review articles, (2) studies without the raw data of the XRCC3 genotype, (3) repeatedly published data.

\section{Data extraction}

Both investigators extracted the information independently including first author, journal, year of publication, country of origin, cancer type, genotyping method, sample size, source of control groups, frequency of genotypes and alleles in cases and controls, and the Hardy-Weinberg equilibrium (HWE) of genotype distribution in controls. . If authors generated different results, they would check the data again and have a discussion to come to an agreement.

\section{Statistical analyses}

Meta-analysis was performed using the CMA 5.0 (Copenhagen, 2008). The risk of CRC associated with the XRCC3 Thr241Met polymorphism was estimated for each study by odds ratio (OR) and $95 \%$ confidence interval $(95 \% \mathrm{CI})$.

All $\mathrm{P}$ values in this study were two-sided, and $\mathrm{p}=0.05$ was set as the threshold value for statistical significance. We used the following models to calculate different ORs: the allele model (A vs . a), the additive genetic model (AA $v s$. aa), the dominant genetic model (AA+Aa vs. aa), and the recessive genetic model (AA vs. Aa+aa). If the $\mathrm{P}$ value was greater than 0.100 according to the $\mathrm{Q}$-test, indicating a lack of heterogeneity among studies, the summary OR estimate of each study was calculated by a fixed-effects model (the Mantel-Haenszel method). Otherwise, the random-effects model (the DerSimonian-Laird method) was performed. Heterogeneity was estimated with the Cochran's Q-statistic, and p=0.05 was considered to be an indication of statistically significant heterogeneity. Also, effect of the heterogeneity quantified with the $\mathrm{I}^{2}$ test (Huedo-Medina TB et al., 2006). As a guide, $\mathrm{I}^{2}$ values ranged from 0 to $100 \%$, and values of $25 \%, 50 \%$, and $75 \%$ were considered to represent low, moderate, and high levels of heterogeneity, respectively. The funnel plot was drawn to assess publication biases. The test suggested was used to test the funnel-plot symmetry. This test involved building a regression model, in which the standardized estimate of the size effect was the dependent variable, and the inverse of the standard error was the independent variable. If the intercept was significantly different from zero, the estimate of the effect was considered biased. The significance of the pooled OR was determined with the $\mathrm{Z}$ test. Each study was removed in turn for sensitivity analyses, and the remaining studies were reanalyzed to assess the stability of results. Subgroup and metaregression analyses were performed to investigate potential sources of heterogeneity.

\section{Results}

\section{Meta-analysis XRCC3 polymorphism}

The meta-analysis of the association between the XRCC3 Thr241Met polymorphism and colorectal cancer consisted of 16 studies, which included 5193 cases and 6645 controls. It is presented in Table1. The distributions of genotypes among controls of the whole studies conformed to HWE. That there was a significant difference between XRCC3 Thr241Met polymorphism and CRC risk in the overall 12 studies only under dominant genetic model (CC+CT vs. TT: OR 0.575, 95\%CI 0.498-1.665, $\mathrm{P}<0.001$, Pheterogeneity $=0.00, \mathrm{I}^{2}=83 \%$ ). The forest plot of dominant model was showed in Figure 1. Overall, no significant association was found between XRCC3 polymorphism and colorectal cancer risk in rest of genetic models (Allele model: C vs. T: OR=0.991,95\%=CI 0.931$1.055, \mathrm{p}=0.77$, Pheterogeneity $=0.00, \mathrm{I}^{2}=92 \%$; Recessive model: $\mathrm{CC} v s$. $\mathrm{CT}+\mathrm{TT}: \mathrm{OR}=1.059,95 \% \mathrm{CI}=0.975-1.150$, 
The XRCC3 Thr241Met Polymorphism and Risk of Colorectal Cancer: a Meta Analysis Table 1. Characteristics of Studies Included in the Meta-Analysis

\begin{tabular}{|c|c|c|c|c|c|c|c|c|c|c|c|c|c|}
\hline \multirow[t]{3}{*}{ Authors } & \multirow[t]{3}{*}{ Country } & \multirow[t]{3}{*}{ Ethnicity } & \multirow{3}{*}{$\begin{array}{c}\text { Case/control } \\
\text { No }\end{array}$} & \multicolumn{5}{|c|}{ CRC Patients } & \multicolumn{5}{|c|}{ Controls } \\
\hline & & & & \multicolumn{3}{|c|}{ Genotypes } & \multicolumn{2}{|c|}{ Alleles } & \multicolumn{3}{|c|}{ Genotypes } & \multicolumn{2}{|c|}{ Alleles } \\
\hline & & & & $\mathrm{CC}$ & $\mathrm{CT}$ & TT & $\mathrm{C}$ & $\overline{\mathrm{T}}$ & $\overline{\mathrm{CC}}$ & $\mathrm{CT}$ & $\overline{\mathrm{TT}}$ & $\mathrm{C}$ & $\mathrm{T}$ \\
\hline $\begin{array}{l}\text { Nissar et al. } \\
2014\end{array}$ & Kashmir & Asian & $120 / 150$ & 72 & 34 & 14 & 178 & 62 & 118 & 22 & 10 & 258 & 42 \\
\hline $\begin{array}{l}\text { Zhao et al. } \\
2012\end{array}$ & China & Asian & $485 / 970$ & 357 & 89 & 38 & 803 & 165 & 846 & 81 & 43 & 1773 & 167 \\
\hline $\begin{array}{l}\text { Krupa et al. } \\
2011\end{array}$ & Poland & Caucasian & $100 / 100$ & 36 & 55 & 9 & 127 & 73 & 50 & 47 & 3 & 147 & 53 \\
\hline $\begin{array}{l}\text { Moghtit et al. } \\
2014\end{array}$ & Algeria & Caucasian & $129 / 148$ & 45 & 68 & 16 & 158 & 100 & 55 & 72 & 21 & 182 & 114 \\
\hline $\begin{array}{l}\text { Improta et al. } \\
2008\end{array}$ & Italy & Caucasian & $109 / 121$ & 40 & 43 & 26 & 123 & 95 & 67 & 46 & 8 & 180 & 62 \\
\hline $\begin{array}{l}\text { Mucha et al. } \\
2013\end{array}$ & Poland & Caucasian & $194 / 209$ & 97 & 72 & 25 & 266 & 112 & 80 & 104 & 25 & 264 & 154 \\
\hline Gil et al. 2012 & Poland & Caucasian & $132 / 100$ & 55 & 65 & 12 & 175 & 89 & 51 & 36 & 13 & 138 & 62 \\
\hline $\begin{array}{l}\text { Yeh et al. } \\
2005\end{array}$ & Taiwan & Asian & $721 / 734$ & 660 & 60 & 1 & 1380 & 62 & 658 & 74 & 2 & 1390 & 78 \\
\hline $\begin{array}{l}\text { Canbay et al. } \\
2011\end{array}$ & Turkey & Caucasian & $79 / 247$ & 23 & 45 & 11 & 91 & 67 & 74 & 146 & 27 & 294 & 200 \\
\hline $\begin{array}{l}\text { Wang et al. } \\
2010\end{array}$ & India & Asian & $302 / 291$ & 213 & 79 & 10 & 505 & 99 & 197 & 85 & 9 & 479 & 103 \\
\hline $\begin{array}{l}\text { Curtin et al. } \\
2009\end{array}$ & USA & Caucasian & $1581 / 1948$ & 671 & 702 & 208 & 2044 & 1118 & 760 & 911 & 277 & 2431 & 1465 \\
\hline $\begin{array}{l}\text { Pardini et al. } \\
2008\end{array}$ & Czech & Caucasian & $532 / 532$ & 203 & 264 & 65 & 724 & 394 & 219 & 250 & 63 & 688 & 376 \\
\hline $\begin{array}{l}\text { Moreno et al. } \\
2006\end{array}$ & Spain & Caucasian & $361 / 316$ & 140 & 170 & 51 & 450 & 272 & 111 & 158 & 47 & 380 & 252 \\
\hline $\begin{array}{l}\text { Skjelbred et } \\
\text { al. } 2006\end{array}$ & Norwegian & Caucasian & $157 / 399$ & 64 & 73 & 20 & 201 & 113 & 138 & 201 & 60 & 477 & 321 \\
\hline Jin et al. 2005 & China & Asian & $140 / 280$ & 124 & 15 & 1 & 263 & 17 & 268 & 11 & 1 & 547 & 13 \\
\hline $\begin{array}{l}\text { Krupa and } \\
\text { Blasiak et al. } \\
2004\end{array}$ & Poland & Caucasian & $51 / 100$ & 1 & 27 & 23 & 29 & 73 & 11 & 81 & 8 & 103 & 97 \\
\hline
\end{tabular}

$\mathrm{p}=0.174$, Pheterogeneity $=0.00, \mathrm{I}^{2}=83 \%$; Additive genetic model: CC vs. TT: OR $=0.942,95 \% \mathrm{CI}=0.822-1.080$, $\mathrm{p}=0.392$, Pheterogeneity $=0.00, \mathrm{I}^{2}=67 \%$; Heterozygote genetic model: CT vs. TT: OR=0.947, 95\%CI $=0.827$ $1.084, \mathrm{p}=0.431$, Pheterogeneity $=0.002, \mathrm{I}^{2}=57 \%$, Figure 1 ).

\section{Subgroup analysis}

There were an insufficient number of studies that reported CRC risk by sex; however, subgroups, by ethnicity were performed. There were an insufficient number of studies from the Asia to include to the subgroup analysis. It turned out that the XRCC3 Thr241Met polymorphism was associated with a significantly increased CRC risk in Asians rather than in Caucasian.

\section{Caucasian}

The meta-analysis of the association between the XRCC3 Thr241Met polymorphism and CRC in Caucasian consisted of 12 studies, which included 3545 cases and 4370 controls. In the overall analysis, a significant association between XRCC3 polymorphism and CRC risk was found under Heterozygote genetic model (CT vs. TT: $\mathrm{OR}=0.929,95 \% \mathrm{CI}=0.806-1.070, \mathrm{p}=0.308$, Pheterogeneity $\left.=0.002, \mathrm{I}^{2}=57 \%\right)$. The forest plot of 4 genetic models was showed in Figure 1. Dominant model: $\mathrm{CC}+\mathrm{CT} v s$. TT: $\mathrm{OR}=0.947,95 \% \mathrm{CI}=0.828-0.902$, $\mathrm{p}=1.083$, Pheterogeneity $=0.426, \mathrm{I}^{2}=74 \%$; Allele model: C vs. T: OR=1.055, $95 \%=$ CI $0.986-1.29, \mathrm{p}=0.123$, Pheterogeneity $=0.00, \mathrm{I}^{2}=92 \%$; Recessive model: $\mathrm{CC}$ vs. CT+TT: $\mathrm{OR}=0.981,95 \% \mathrm{CI}=0.894-1.077, \mathrm{p}=0.69$, Pheterogeneity $=0.00, \mathrm{I}^{2}=83 \%$; Additive genetic model: CC vs. TT: OR $=1.008,95 \% \mathrm{CI}=0.872-1.166, \mathrm{p}=0.909$, Pheterogeneity $=0.00, \mathrm{I}^{2}=67 \%$.

\section{Asian}

Four studies evaluated the XRCC3 Thr241Met polymorphism and CRC risk in Asians. It was found that the dominant and Allele genetic models of the XRCC3 Thr241Met polymorphism were significantly correlated with increasing risk of CRC (Dominant model: CC+CT vs. TT: $\mathrm{OR}=0.609,95 \% \mathrm{CI}=411-0.902, \mathrm{p}=0.013$, Pheterogeneity $=0.54, \mathrm{I}^{2}=0.00 \%$; Allele model: $\mathrm{C} v s$. T: 


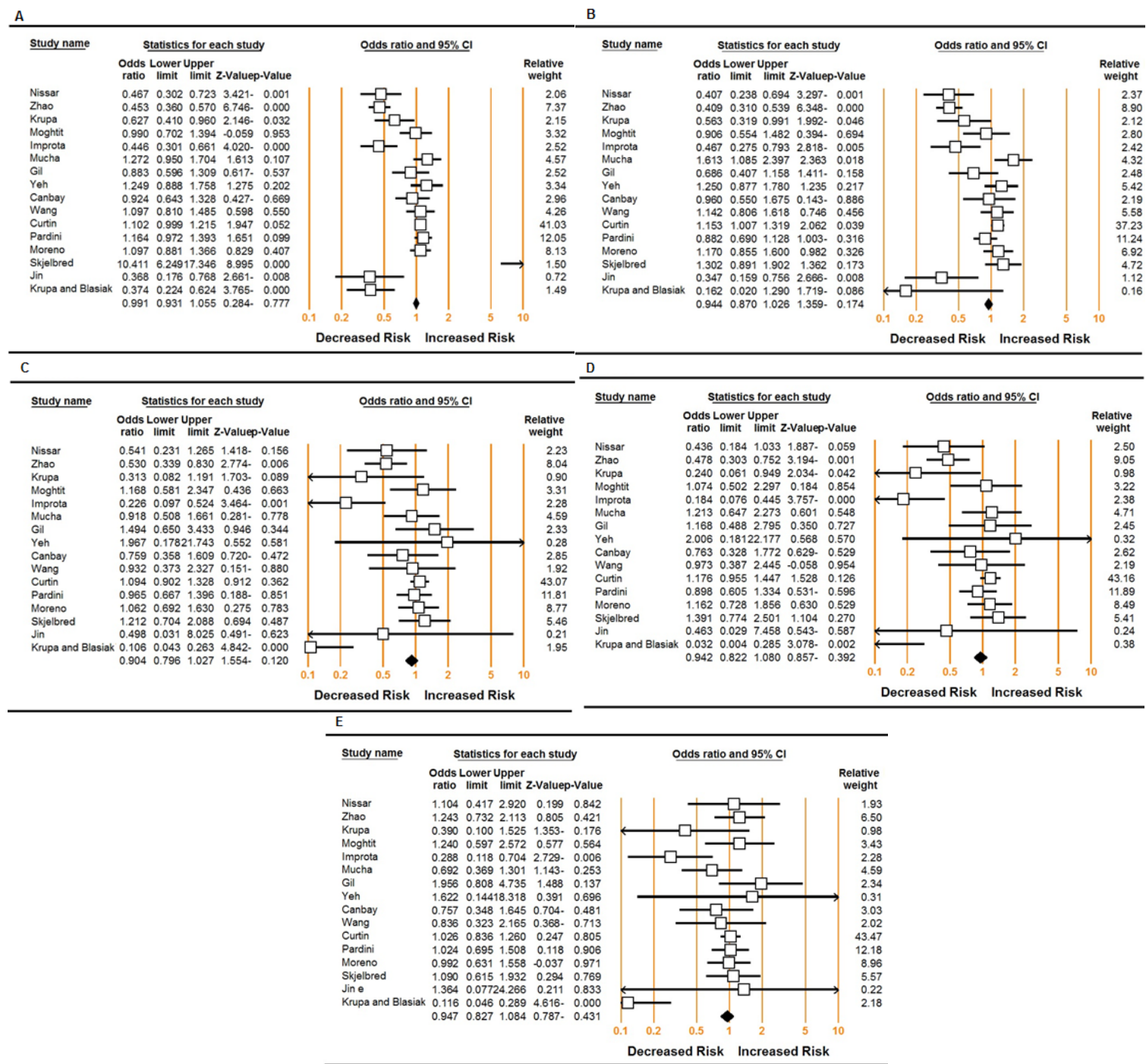

Figure 1. Meta-analysis of the Association between XRCC3 Polymorphism and Susceptibility to Colorectal Cancer (A: Cvs.T; B: CCvs.CT+TT; C: CC+CTvs.TT; D: CCvs.TT; E: CTvs.TT)
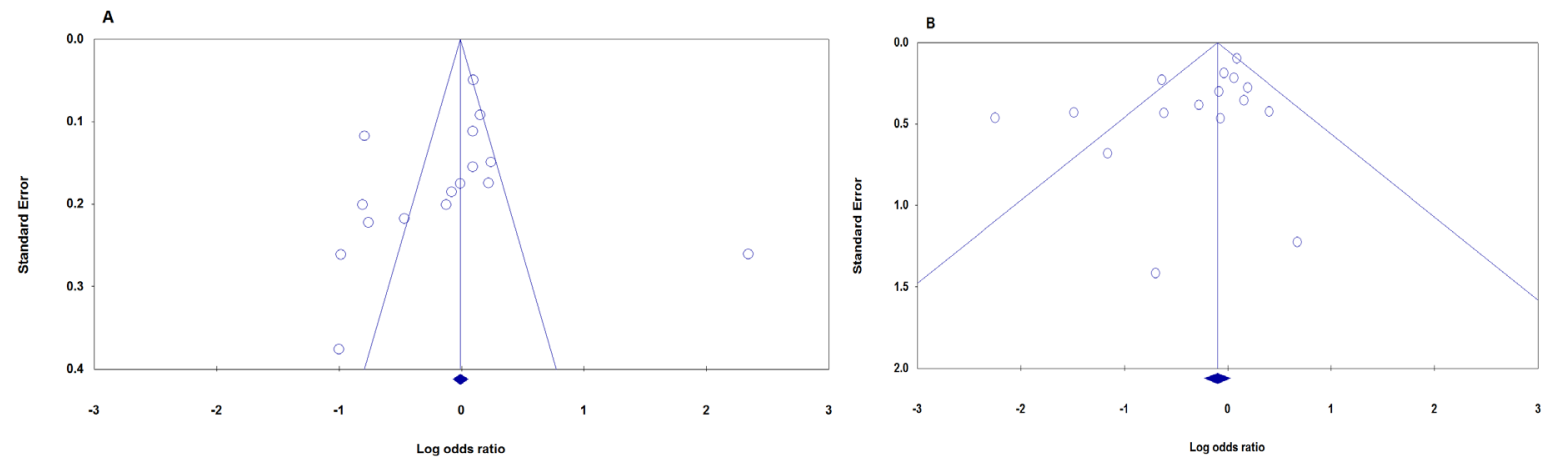

Figure 2. Begg's Funnel Plot of the Meta-Analysis of Colorectal Cancer Risk and XRCC3 Polymorphism (A: Allel model and B: dominant model)

$\mathrm{OR}=0.708,95 \%=\mathrm{CI} 0.605-0.829, \mathrm{p}=0.000$, Pheterogeneity $\left.=0.000, \mathrm{I}^{2}=92 \%\right)$. However, no significant effect of XRCC3 Thr241Met polymorphism on the CRC susceptibility was found in Asians for 4 other genetic models (Recessive model: $\mathrm{CC} v s$. CT+TT: OR=1.408,95\%CI $=1.177-1.685$, $\mathrm{p}=0.00$, Pheterogeneity $=0.00, \mathrm{I}^{2}=83 \%$; Additive genetic model: CC vs. TT: OR $=0.566,95 \% \mathrm{CI}=0.381-0.843$, $\mathrm{p}=0.005$, Pheterogeneity $=0.00, \mathrm{I}^{2}=67 \%$; Heterozygote genetic model: CT vs. TT: $\mathrm{OR}=1.151,95 \% \mathrm{CI}=0.7351-$ $1.804, \mathrm{p}=0.539$, Pheterogeneity $=0.002, \mathrm{I}^{2}=57 \%$ ).

\section{Heterogeneity and sensitivity analyses}

Heterogeneity may be due to the presence of one or two outlying studies with results that conflict with the rest of the studies. Subsequent sensitivity analyses were performed by removing the individual studies sequentially. 
The exclusion of each study failed to resolve the heterogeneity across the remaining studies. The observed heterogeneity is likely due to misclassification, because some studies used a laboratory method that may not accurately distinguish between the alleles of the XRCC3 Thr241Met polymorphism.

\section{Publication bias}

Begger's funnel plot and Egger's linear regression test were performed to assess the publication biases of the selected studies. The shape of the funnel plot for publication bias appeared to be symmetrical, although there was some uncertainty regarding the degree of symmetry. The Egger's test results and Begg's funnel plot (Figure 2) suggested no evidence of publication bias in the meta-analysis of XRCC3 only for allele model $(p=0.0 .216)$, although possible publication bias was suggested for dominant model $(\mathrm{p}=0.041)$, recessive model $(\mathrm{p}=0.042)$, additive model $(\mathrm{p}=0.022)$ and heterozygote model $(\mathrm{p}=0.022)$.

\section{Discussion}

The XRCC3 Thr241Met polymorphism exerts different effect on diverse types of cancers and even the same cancer in different populations, indicating the underlying complicated mechanisms in tumorigenesis (Chen et al., 2014). A previous meta analysis by Sun HM et al. has revealed that the XRCC3 Thr241Met polymorphism is weakly associated with the risk of bladder cancer, but not lung cancer (Sun et al., 2010).

It is well documented that the genetic polymorphism may contribute to susceptibility to cancer. Although a number of previous studies have reported a significant association between the XRCC3 Thr241Met polymorphism and $\mathrm{CRC}$ risk, others have identified no such association (Fasching et al., 2009). In order to resolve this conflict, in the current study, a meta-analysis was conducted to examine the associa $\neg$ tion between a commonly studied XRCC3 Thr241Met polymorphism and CRC risk. A total of 5,193 CRC cases and 6,645 controls from 16 studies were included in the final analysis, to derive a more precise estimation of the presence or absence of this association. The results revealed that no significant association was observed between XRCC3 Thr241Met polymorphism and risk of CRC. This reduced risk of CRC for the TT genotype was not supported by all of the included studies. In several individual studies, the TT genotype was associated with an increased risk of CRC (Liu et al., 2013).

In all studies, people with different nationalities have different genotype frequencies; however, in the stratified analysis by ethnicity, a significant association of the XRCC3 Thr241Met polymorphism with CRC risk in the Asians but not the Caucasians was demonstrated, in consistence to the finding of Liu L., et al (Liu et al., 2013). The discrepancy may be due to the different genotype penetrance of XRCC3 Thr241Met in different ethnicities (Shizhong Han et al., 2006). Thus far, little has been known about the potential causes needing further investigation. In addition, some of the studies conducted in Asian countries had a very small sample size and did not have adequate power to detect the possible risk for XRCC3 Thr241Met polymorphism, and the observed significant ORs in these studies may be false associations (Wang et al., 2010; Nissar et al., 2014). Also, most of the studies have shown that the polymorphism of XRCC3 Thr241Met to contribute to the development of CRC across Caucasian ethnicity countries in many previous individual studies (Improta et al., 2008; Krupa et al., 2011). To clarifying such findings, the quality of study design is of great importance. However, some of the analyzed studies had methodological shortcomings. For instance, the source and the selection criteria of case and/or control groups were not clearly presented in some studies leading to possible biases. These negative results suggest that the evidence to evaluate this SNPs associated with risk of CRC are insufficient, more large sample size and welldesigned researches about the associations are warranted to further confirm the results.

Many of the studies not incorporated both men and women into the case control groups. However, far fewer studies stratified their results based on sex. Of the 16 studies included in this meta analysis only a few studies reported significant OR based on sex and genotype, which was contrary to the summary results in this meta-analysis.

Heterogeneity is to be expected in a meta-analysis (Higgins et al., 2008). Studying heterogeneity was a considerable problem in our meta-analysis. The heterogeneity existed in allelic genetic model, heterozygote model, and dominant genetic model in overall analysis. The meta regression analysis did not identify the source of heterogeneity. In sensitivity analysis, heterogeneity reduced after one literature was excluded, but the pooled ORs did not alter remarkably, implying that the results were statistically stable and reliable. The Begg funnel plot and Egger's test were also negative for publication bias.

Some limitations of this meta-analysis should be addressed. First, the number of published studies was not sufficiently large for a comprehensive analysis, and some studies with small size may not have enough statistical power to explore the real association. The small sample size was not ideal for detecting small genetic effects. Secondly, we were not able to address the sources of heterogeneity that existed among studies for each polymorphism. Finally, we could not perform further subgroup stratification analysis because of the limited number of published studies and data. In spite of these limitations, our meta- analysis had several advantages. First, substantial number of cases and controls were pooled from different studies, which significantly increased statistical power of the analysis. Second, the quality of case-control studies included in current meta-analysis was satisfactory and met our inclusion criterion.

The studies included in the meta-analysis were conducted in fifteen different countries. This is potentially both a strength and weakness of our analysis. The potential weakness rests with the increased heterogeneity of some of the results. In conclusion, the results of this metaanalysis suggest that the XRCC3 Thr241Met genotype is associated with a reduced risk of CRC in Caucasians. 


\section{Acknowledgements}

This work was supported by the Shahid Sadoughi Medical Science University.

\section{References}

Alanazi M, Pathan AA, Ajaj SA, et al (2013). DNA Repair Genes XRCC1, XRCC3, XPD, and OGG1 Polymorphisms among the Central Region Population of Saudi Arabia. Biol Res, 46, 161-7.

Arafa MA, Sallam S, Jriesat S, et al (2011). Colorectal cancer screening amongst first degree relatives of colon cancer cases in Jordan. Asian Pac J Cancer Prev, 12, 1007-11.

Bewick MA, Conlon MS, Lafrenie RM, et al (2006). Polymorphisms in XRCC1, XRCC3, and CCND1 and survival after treatment for metastatic breast cancer. J Clin Oncol, 24, 5645-51.

Brenneman MA, Wagener BM, Miller CA, et al (2002). XRCC3 controls the fidelity of homologous recombination: roles for XRCC3 in late stages of recombination. Mol Cell, 10, 387-95.

Canbay E, Cakmakoglu B, Zeybek U, Sozen S (2011). Association of APE1 and hOGG1 polymorphisms with colorectal cancer risk in a Turkish population. Curr Med Res Opin, 27, 1295-302.

Chen X, Wang Z, Yan Y, et al (2014). XRCC3 C18067T polymorphism contributes a decreased risk to both basal cell carcinoma and squamous cell carcinoma: evidence from a meta-analysi. PLoS One, 15, 84195.

Coory MD (2010). Comment on: Heterogeneity in meta-analysis should be expected and appropriately quantified. Int $J$ Epidemiol, 39, 932-3.

Curtin K, Samowitz WS, Wolff RK, et al (2009). Assessing tumor mutations to gain insight into base excision repair sequence polymorphisms and smoking incolon cancer. Cancer Epidemiol Biomarkers Prev, 18, 3384-8.

Fasching PA, Gayther S, Pearce L, et al (2009). Role of genetic polymorphisms and ovarian cancer susceptibility. Mol Oncol, 3,171-81.

Gil J, Ramsey D, Stembalska A, et al (2012). The C/A polymorphism in intron 11 of the XPC gene plays a crucial role in the modulation of an individual's susceptibility to sporadic colorectal cancer. Mol Biol Rep, 39, 527-34.

He XF, Wei W, Li JL, et al (2013). Association between the XRCC3 T241M polymorphism and risk of cancer: evidence from 157 case-control studies. Gene, 523, 10-9.

Higgins JP (2008). Commentary: Heterogeneity in meta-analysis should be expected and appropriately quantified. Int $J$ Epidemiol, 37, 1158-60.

Huedo-Medina TB, Sanchez-Meca J, Marin-Martinez F, Botella J (2006). Assessing heterogeneity in meta-analysis: Q statistic or $\mathrm{I}^{2}$ index? Psychol Methods, 11, 193-206.

Improta G, Sgambato A, Bianchino G, et al (2008). Polymorphisms of the DNA repair genes XRCC1 and XRCC3 and risk of lung and colorectal cancer: a case-control study in a Southern Italian population. Anticancer Res, 28, 2941-6.

Jemal A, Bray F, Center MM, Ferlay J, et al (2011). Global cancer statistics. CA Cancer J Clin, 61, 69-90.

Jin MJ, Chen K, Song L, et al (2005). The association of the DNA repair gene XRCC3 Thr241Met polymorphism with susceptibility to colorectal cancer in a Chinese population. Cancer Genet Cytogenet, 163, 38-43.

Gao CM, Ding JH, Li SP, et al (2014). Polymorphisms in XRCC1 gene, alcohol drinking, and risk of colorectal cancer: a casecontrol study in Jiangsu Province of China. Asian Pac J Cancer Prev, 14, 6613-8.
Krupa R, Sliwinski T, Wisniewska-Jarosinska M, et al (2011). Polymorphisms in RAD51, XRCC2 and XRCC3 genes of the homologous recombination repair in colorectal cancer--a case control study. Mol Biol Rep, 38, 2849-54.

Krupa R, Blasiak J (2004). An association of polymorphism of DNA repairs genes XRCC1 and XRCC3 with colorectal cancer. J Exp Clin Cancer Res, 23, 285-94.

Mahmoudi T, Karimi K, Arkani M, et al (2014). Parathyroid hormone gene rs6256 and calcium sensing receptor gene rs1801725 variants are not associated with susceptibility to colorectal cancer in Iran. Asian Pac J Cancer Prev, 15, 6035-9.

Moghtit FZ, Aberkane MS, Le Morvan V, et al (2014). No association between XRCC3 Thr241Met and XPD Lys751Gln polymorphisms and the risk of colorectal cancer in West Algerian population: a case-control study. Med Oncol, 31, 942.

Moreno V, Gemignani F, Landi S, et al (2014). Polymorphisms in genes of nucleotide and base excision repair: risk and prognosis of colorectal cancer. Clin Cancer Res, 12, 2101-8.

Mucha B, Przybylowska-Sygut K, Dziki AJ, et al (2013). Association of Thr241Met polymorphism of XRCC3 gene with risk of colorectal cancer in the Polish population. Pol J Pathol, 64, 185-90.

Nissar S, Sameer AS, Lone TA, et al (2014). XRCC3 thr241met gene polymorphism and risk of colorectal cancer in Kashmir: a case control study. Asian Pac J Cancer Prev, 15, 9621-5.

Liu L, Miao L, Ji G, , et al (2013). Association between XRCC1 and XRCC3 polymorphisms and colorectal cancer risk: a meta-analysis of 23 case-control studies. Mol Biol Rep, 40, 3943-52.

Pardini B, Naccarati A, Novotny J, et al (2008). DNA repair genetic polymorphisms and risk of colorectal cancer in the Czech Republic. Mutat Res, 638, 46-53.

Shizhong Han, Hong-Tao Zhang, Zhentian Wang, et al (2006). DNA repair gene XRCC3 polymorphisms and cancer risk: a meta-analysis of 48 case-control studies. Eur J Human Genetics, 14, 1136-44.

Skjelbred CF, Saebø M, Wallin H, et al (2006). Polymorphisms of the XRCC1, XRCC 3 and XPD genes and risk of colorectal adenoma and carcinoma, in a Norwegian cohort: a case control study. BMC Cancer, 16, 6-67.

Sun H, Qiao Y, Zhang X, et al (2010). XRCC3 Thr241Met polymorphism with lung cancer and bladder cancer: a metaanalysis. Cancer Sci, 101, 1777-82.

Tebbs RS, Zhao Y, Tucker JD, et al (1995), Thompson LH. Correction of chromosomal instability and sensitivity to diverse mutagens by a cloned cDNA of the XRCC3 DNArepair gene. Proc Natl Acad Sci USA, 92, 6354-8.

Wang J, Zhao Y, Jiang J, et al (2010). Polymorphisms in DNA repair genes XRCC1, XRCC3 and XPD, and colorectal cancer risk: a case-control study in an Indian population. $J$ Cancer Res Clin Oncol, 136, 1517-25.

Yeh CC, Sung FC, Tang R, et al (2005). Polymorphisms of the XRCC1, XRCC3, \&amp; XPD genes, and colorectal cancer risk: a case-control study in Taiwan. BMC Cancer, 5, 12.

Zhou CP, Pan HZ, Li FX, et al (2014). Association analysis of colorectal cancer susceptibility variants with gastric cancer in a ChineseHan population. Genet Mol Res, 13, 3673-80.

Zhan P, Wang Q, Qian Q, Yu LK (2013). XRCC3 Thr241Met gene polymorphisms and lung cancer risk: a meta-analysis. $J$ Exp Clin Cancer Res, 32, 1 .

Zhao Y, Deng X, Wang Z, et al (2012). Genetic polymorphisms of DNA repair genes XRCC1 and XRCC3 and risk of colorectal cancer in Chinese population. Asian Pac J Cancer Prev, 13, 665-9. 